\title{
NATURAL EXTENSIONS OF PROBABILITY MEASURE IN FUNCTION SPACE
}

\author{
R. Z. YEH \\ (Received 21 December 1971; revised 25 March 1974) \\ Communicated by C. C. Heyde
}

\section{Background}

Let $\left\{X_{t}\right\}_{t \in T}$ be a family of real $(R)$ random variables defined on a probability space $(\Omega, \mathscr{A}, P)$ and having the ranges in a subset $S$ of $R$, that is, $X_{t}(\Omega) \subset S$ for all $t$. Let $X$ be the mapping of $\Omega$ into the function space $S^{T}$

$$
X: \Omega \rightarrow S^{T}
$$

such that for any $\omega \in \Omega$

$$
[X(\omega)](t)=X_{t}(\omega)
$$

We shall write $X=\left\{X_{t}\right\}_{t \in T}$ and call $X$ the random function arising from $\left\{X_{t}\right\}_{t \in T}$. It is well-known that any finite subfamily of $\left\{X_{t}\right\}_{t \in T}$ induces a "finite joint distribution" in $S^{T}$, and according to Kolmogorov (1933) these finite joint distributions can be simultaneously extended to a probability measure $P_{0}$ on the Borel class $\mathscr{B}_{0}$ of subsets of $S^{T}$. This extension is natural in the sense that for any $B \in \mathscr{B}_{0}$ $P_{0}(B)$ turns out to be exactly $P\left[X^{-1}(B)\right]$. The Kolmogorov extension $P_{0}$ has however a shortcoming in that its domain $\mathscr{B}_{0}$ is not broad enough to include many events of practical interest.

Following Kakutani (1943), Nelson (1959) has formulated a regular Borel measure $P_{1}$, which extends the Kolmogorov extension $P_{9}$ to the topological Borel class $\mathscr{B}_{1}$ containing $\mathscr{B}_{0}$ provided that $S$ is a compact subset of $R$. The Kakutani extension $P_{1}$ has the following regularity property: for any $B \in \mathscr{B}_{1}$

$$
\sup _{F \subset B} P_{1}(F)=P_{1}(B)=\inf _{G \supset B} P_{1}(G)
$$

where $F$ and $G$ respectively denote closed and open subsets of $S^{T}$. The definitions of $\mathscr{B}_{1}$ and $\mathscr{B}_{0}$ are given in section 2.

The purpose of this paper is to show by a simple well-known example of Doob (1953) that the Kakutani extension is not natural in that given $B \in \mathscr{B}_{1} P_{1}(B)$ 
may not be the same as $P\left[X^{-1}(B)\right]$. In contrast the naturalness of Doob's extension (1937) will be revealed by a suitable formulation.

\section{Doob's example}

Let $(\Omega, \mathscr{A}, P)$ be the probability space where $\Omega=[0,1], \mathscr{A}$ is the Borel subsets of $[0,1]$, and $P$ is the Lebesgue measure. Let $T=[0,1]$, and for each $t \in[0,1]$ let $X_{t}: \Omega \rightarrow R$ be defined by $X_{t}(\omega)=\delta_{t \omega}$ (Kronecker delta taking the value 1 if $\omega=t$ and 0 if $\omega \neq t$ ). Clearly, the resulting random function $X$ takes $\omega$ to a function on the interval $[0,1]$ which assumes 0 everywhere except at $t=\omega$, where it assumes 1 . Thus, $X: \Omega \rightarrow S^{T}$ with $S=[0,1]$, a compact subset of $R$.

It is not difficult to see that all the finite joint distributions induced by $X$ are one-point distributions concentrated at the origins of finite dimensional Euclidean spaces. According to Nelson (1959) these finite joint distributions can now be extended to the regular Borel measyre $P_{1}$, whose domain is the topological Borel class $\mathscr{B}_{1}$, which is the sigma-algebra of subsets of $S^{T}$ generated by the topology (that is, all the open sets) generated by the (open) neighborhoods of the form

$$
N=\left\{x \in S^{T}: a_{i}<x\left(t_{i}\right)<b_{i}\right\}
$$

where $t_{i} \in T$, and $-\infty \leqq a_{i}<b_{i}<\infty$ are pairs of real numbers for $i=1,2, \cdots, n$ with $n$ finite but otherwise arbitrary. The Borel class $\mathscr{B}_{0}$ is simply the sigmaalgebra of subsets of $S^{T}$ generated by the subsets $N$ described above. Clearly, $\mathscr{B}_{1} \supset \mathscr{B}_{0}$ since $\mathscr{B}_{1}$ is generated by a larger collection of subsets of $S^{T}$.

\section{Unnaturalness of Kakutani extension}

The unnaturalness of $P_{1}$ is shown in regard to the simple Doob process described above. Specifically, we will show that the elementary event $\{\theta\}$ where $\theta$ is the zero function in $S^{T}$, that is, $\theta(t) \equiv 0$, receives $P_{1}$ measure 1 while $X^{-1}(\{\theta\})$ being the empty subset of $\Omega$ receives $P$ measure 0 .

It suffices to show that every open set containing $\{\theta\}$ has $P_{1}$ measure 1 , for then by the regularity of $P_{1} P_{1}(\{\theta\})$ must be 1 . Let $G$ be any open subset of $S^{T}$ containing $\{\theta\}$, then there must be an open neighborhood $N$ containing $\theta$ contained in $G, \theta \in N \subset G$. Now to contain $\theta, N$ must be of the form

$$
N=\left\{x \in S^{T}: a_{i}<x\left(t_{i}\right)<b_{i}\right\}
$$

where $a_{\imath}<0<b_{i}$ for $i=1,2, \cdots, n$. But since each finite joint distribution is concentrated at the origin, this means $P_{1}(N)=1$; and consequently $P_{1}(G)=1$, completing the proof that

for $B=\{\theta\}$.

$$
P_{1}(B) \neq P\left[X^{-1}(B)\right]
$$




\section{Formulation of Doob extension}

Let $\left\{X_{t}\right\}_{t \in T}$ be a family of real random variables on $(\Omega, \mathscr{A}, P)$ constituting the random function $X: \Omega \rightarrow R^{T}$ (see section 1), and let $\mathscr{X}=X(\Omega)$ be the range of the random function $X$, then we have the following

Proposition 1. The Kolmogorov outer measure of $\mathscr{X}$ is 1 , that is,

$$
\bar{P}_{0}(\mathscr{X})=\inf _{B \supset \mathscr{X}} P_{0}(B)=1
$$

where $B$ are members of $\mathscr{B}_{0}$.

Proof. In view of naturalness of Kolmogorov extension $P_{0}$ we have for any $B \supset \mathscr{X}$ and $B \in \mathscr{B}_{0}$

$$
P_{0}(B)=P\left[X^{-1}(B)\right]=P(\Omega)=1
$$

and hence $\bar{P}_{0}(\mathscr{X})=1$.

Definition 1. By the Doob class $\mathscr{B}_{\mathscr{X}}$ of subsets of $R^{T}$ relatibve to $\mathscr{X} \subset R^{T}$ we mean the totality of subsets of $R^{T}$ of the form

$$
D=(B \cap \mathscr{X}) \cup H
$$

where $B$ belongs to the Borel class $\mathscr{B}_{0}$ and $H$ is a subset of $\mathscr{X}^{c}=R^{T}-\mathscr{X}$.

Proposition 2. A Doob class $\mathscr{B}_{\mathscr{x}}$ relative to any $\mathscr{X}$ is a sigma-algebra containing the Borel class $\mathscr{B}_{0}$.

PROOF. To see $\mathscr{B}_{\mathscr{X}} \supset \mathscr{B}_{0}$ it suffices to show that any $B \in \mathscr{B}_{0}$ can be expressed in the form

$$
B=(B \cap \mathscr{X}) \cup\left(B \cap \mathscr{X}^{c}\right)
$$

That $\mathscr{B}_{x}$ is a sigma-algebra is just as obvious.

Definition 2. We define the Doob extension $P_{\mathscr{x}}$ of Kolmogorov extension $P_{0}$ of finite joint distributions for $R^{T}$ as follows: for any $D \in \mathscr{B}_{\mathscr{X}}$

$$
P_{\mathscr{X}}(D)=P_{0}(B)
$$

where $D=(B \cap \mathscr{X}) \cup H$ with $B \in \mathscr{B}_{0}$ and $H \subset \mathscr{X}$.

Obviously $P_{x}$ is an extension of $P_{0}$ since for any $B \in \mathscr{B}_{0}$ we have $B=(B \cap \mathscr{X}) \cup\left(B \cap \mathscr{X}^{c}\right)$ and $P_{\mathscr{X}}(B)=P_{0}(B)$. To see that $P_{\mathscr{X}}$ is well-defined we must show the following

Proposition 3. If $D=\left(B^{\prime} \cap \mathscr{X}\right) \cup H^{\prime}=\left(B^{\prime \prime} \cap \mathscr{X}\right) \cup H^{\prime \prime}$, then

$$
P_{0}\left(B^{\prime}\right)=P_{0}\left(B^{\prime \prime}\right)
$$

Proof. We need only show $P_{0}\left(B^{\prime}-B^{\prime \prime}\right)=P_{0}\left(B^{\prime \prime}-B^{\prime}\right)=0$. We will merely assume $P_{0}\left(B^{\prime}-B^{\prime \prime}\right)>0$ to derive a contradiction, the other assumption leading to a similar contradiction. 
Since by Proposition $1 \inf _{B \supset x} P_{0}(B)=1$, and $\mathscr{B}_{0}$ is a sigma-algebra, there actually exists a $B_{0} \in \mathscr{B}_{0}$ such that $B_{0} \supset \mathscr{X}$ and $P_{0}\left(B_{0}\right)=1$. Now from $H^{\prime}=D-\mathscr{X}=H^{\prime \prime}$ follows $B^{\prime} \cap \mathscr{X}=B^{\prime \prime} \cap \mathscr{X}$ so that $B^{\prime}-B^{\prime \prime} \subset \mathscr{X}^{c}$, therefore

$$
B_{0}-\left(B^{\prime}-B^{\prime \prime}\right) \supset \mathscr{X}
$$

We will derive the contradiction by showing $P_{0}\left[B_{0}-\left(B^{\prime}-B^{\prime \prime}\right)\right]<1$.

Now

but

$$
P_{0}\left[B_{0}-\left(B^{\prime}-B^{\prime \prime}\right)\right]=P_{0}\left(B_{0}\right)-P_{0}\left[B_{0} \cap\left(B^{\prime}-B^{\prime \prime}\right)\right],
$$

and

$$
P_{0}\left[B_{0} \cap\left(B^{\prime}-B^{\prime \prime}\right)\right]=P_{0}\left(B^{\prime}-B^{\prime \prime}\right)-P_{0}\left[B_{0}^{c} \cap\left(B^{\prime}-B^{\prime \prime}\right)\right] \text {, }
$$

$$
P_{0}\left[B_{0}^{c} \cap\left(B^{\prime}-B^{\prime \prime}\right)\right] \leqq P_{0}\left(B_{0}^{c}\right)=1-P_{0}\left(B_{0}\right)=0 ;
$$

therefore $P_{0}\left[B_{0} \cap\left(B^{\prime}-B^{\prime \prime}\right)\right]=P_{0}\left(B^{\prime}-B^{\prime \prime}\right)>0$. Hence

$$
P_{0}\left[B_{0}-\left(B^{\prime}-B^{\prime \prime}\right)\right]<P_{0}\left(B_{0}\right)=1
$$

and the proof is complete.

The following theorem shows the naturalness of Doob extension. Essentially, it is inherited from the naturalness of Kolmogorov extension.

TheOREM. Let $X=\left\{X_{t}\right\}_{t \in T}$ be a real $(R)$ random function defined on a probability space $(\Omega, \mathscr{A}, P)$. Let $\mathscr{X}=X(\Omega) \subset R^{T}$ and $\mathscr{B}_{\mathscr{X}}$ be the Doob class of subsets of $R^{T}$, and let $P_{x}$ be the Doob extension of the Kolmogorov extension $P_{0}$ of finite joint distributions in $R^{T}$. Then for any $D \in \mathscr{B}_{x}, P_{x}(D)=P\left[X^{-1}(D)\right]$.

Proof. Let $D=(B \cap \mathscr{X}) \cup H$, where $B$ is a member of the Borel class $\mathscr{B}_{0}$ and $H \subset \mathscr{X}^{c}$. By Definition 2 and the naturalness of Kolmogorov extension

$$
P_{x}(D)=P_{0}(B)=P\left[X^{-1}(B)\right]
$$

Now

hence

$$
X^{-1}(D)=X^{-1}(B \cap \mathscr{X}) \cup X^{-1}(H)=X^{-1}(B) \cap \Omega \cup \varnothing
$$

and

$$
P\left[X^{-1}(D)\right]=P\left[X^{-1}(B)\right]
$$

$$
P_{x}(D)=P\left[X^{-1}(D)\right]
$$

Finally we add that Doob extension can be strengthened by replacing $\mathscr{X}$ in $\mathscr{B}_{x}$ by $\mathscr{X}_{0}=\mathscr{X}\left(\Omega_{0}\right)$ where $\Omega_{0} \subset \Omega$ with $P\left(\Omega_{0}\right)=1$. This is due to the following

PRoposition 4. Let $\mathscr{X}_{0} \subset \mathscr{X} \subset R^{T}$, then the Doob class $\mathscr{B}_{x_{0}}$ contains the Doob class $\mathscr{B}_{\boldsymbol{X}}$. 
ProOF. To see $\mathscr{B}_{\mathscr{X}} \subset \mathscr{B}_{\mathscr{X}_{0}}$ let $E \in \mathscr{B}_{\mathscr{X}}$, then by Definition $1, E=(B \cap \mathscr{X}) \cup H$ with $B \in \mathscr{B}_{0}$ and $H \subset \mathscr{X}^{c}$. Now

$$
E=\left(B \cap \mathscr{X}_{0}\right) \cup\left[B \cap\left(\mathscr{X}-\mathscr{X}_{0}\right)\right] \cup H
$$

but since $\left[B \cap\left(\mathscr{X}-\mathscr{X}_{0}\right)\right] \cup H$ is clearly contained in $\mathscr{X}_{0}^{c}, \mathrm{t}$ follows that $E \in \mathscr{B}_{x_{0}}$.

\section{References}

J. L. Doob (1953), Stochastic Processes, (John Wiley, New York).

J. L. Doob (1947), 'Probability in function space', Bull. Amer. Math. Soc. 53, 15-30.

J. L. Doob (1937), Stochastic processes depending on continuous parameter, Trans. Amer. Math. Soc. 42, 107-140.

Shizuo Kakutani (1943), Notes on infinite product spaces II, Proc. Imp. Acad. Tokto 19, 184-188.

A. Kolmogorov (1933), (Grundbegriffe der Wahrscheinlichkeitsrechnung, Springer Berlin).

Edward Nelson (1959), Regular probability mesaures on function space, Ann. of Math. (2) 69, 630-643.

University of Hawaii

Honolulu

Hawaii 96822 